Portland State University

PDXScholar

8-1-1999

\title{
Simple Model for Linear and Nonlinear Mixing at Unstable Fluid Interfaces in Spherical Geometry
}

John D. Ramshaw

Portland State University, jdramshaw@yahoo.com

Follow this and additional works at: https://pdxscholar.library.pdx.edu/phy_fac

Part of the Physics Commons

Let us know how access to this document benefits you.

\section{Citation Details}

J.D. Ramshaw, "Simple model for linear and nonlinear mixing at unstable fluid interfaces in spherical geometry," Phys. Rev. E 60, 1775 (1999)

This Article is brought to you for free and open access. It has been accepted for inclusion in Physics Faculty Publications and Presentations by an authorized administrator of PDXScholar. Please contact us if we can make this document more accessible: pdxscholar@pdx.edu. 


\title{
Simple model for linear and nonlinear mixing at unstable fluid interfaces in spherical geometry
}

\author{
John D. Ramshaw \\ Lawrence Livermore National Laboratory, University of California, P.O. Box 808, Livermore, California 94551
}

(Received 5 November 1998; revised manuscript received 6 February 1999)

\begin{abstract}
A simple model was recently described for predicting linear and nonlinear mixing at an unstable planar fluid interface subjected to an arbitrary time-dependent variable acceleration history [J. D. Ramshaw, Phys. Rev. E 58, 5834 (1998)]. Here we present an analogous model for describing the mixing of two adjacent spherical fluid shells of different density resulting from an arbitrary time-dependent mean interface radius $R(t)$. As in the planar case, the model is based on a heuristic expression for the kinetic energy of the system. This expression is based on that for the kinetic energy of a linearly perturbed interface, but with a dynamically renormalized effective wavelength which becomes proportional to the half-width $a(t)$ of the mixing layer in the nonlinear regime. An equation of motion for $s=R^{2} a$ is then derived from Lagrange's equations. This evolution equation properly reduces to Plesset's equation for small perturbations, and to the previous planar model in the limit of very large $R$. The conservation properties of the model are established, and a suitable numerical scheme which preserves these properties is proposed. [S1063-651X(99)13908-4]
\end{abstract}

PACS number(s): 47.20.Bp, 47.20.Ma, 47.27.-i, 47.55.Kf

\section{INTRODUCTION}

There is a continuing current interest in unstable fluid interfaces, particularly those driven by the normal acceleration of adjacent fluid layers with different densities. Most of the work in this area has been restricted to planar interfaces. However, there is also considerable interest in unstable interfaces between adjacent spherical fluid shells, which occur in the implosion of inertial confinement fusion capsules and in certain astrophysical problems. We have previously presented a simple model for describing linear and nonlinear mixing at unstable planar fluid interfaces subjected to an arbitrary time-dependent acceleration history [1]. Our purpose here is to develop an analogous model for the spherical case.

As in the planar case, the present model is based on an analytical expression for the kinetic energy of a linearly perturbed interface, together with a wavelength renormalization hypothesis according to which the effective wavelength of the perturbation becomes proportional to the half-width $a(t)$ of the mixing layer in the nonlinear regime. An equation of motion for $a(t)$ is then obtained from Lagrange's equations, with an additional generalized force term to represent the effects of dissipation [2]. This equation properly reduces to Plesset's equation [3] for a single-mode perturbation in the linear regime with zero dissipation, and to the corresponding planar model [1] in the limit of very large $R$. The development closely parallels that of the planar case [1], with which the reader is assumed to be familiar.

\section{EVALUATION OF THE KINETIC ENERGY}

We consider two adjacent concentric spherical shells of incompressible fluid centered at the origin in a spherical polar coordinate system $(r, \theta, \phi)$. The unperturbed interface between the fluids is located at $r=R(t)$. The inner fluid (fluid 1) has a density $\rho_{1}$ and occupies the region $R_{1}(t)<r$ $<R(t)$, while the outer fluid (fluid 2) has a density $\rho_{2}$ and occupies the region $R(t)<r<R_{2}(t)$, where $R_{1} \ll R \ll R_{2}$.
The unperturbed velocity field within both fluids is purely radial, and is given by $u=\dot{R}(R / r)^{2}$ [3]. We now suppose that the interface location is perturbed to $r=\hat{r}$, where

$$
\hat{r}=\hat{R}(t)+\left(\frac{2 l+1}{2}\right)^{1 / 2} a(t) P_{l}(\cos \theta)
$$

$P_{l}(z)$ is the $l$ th Legendre polynomial $(l \geqslant 1)$, and a normalization factor has been introduced so that $a$ has the same significance as $h$ in the planar case [1], namely, $\sqrt{2}$ times the root-mean-square perturbation height. The perturbation is assumed to be small in the sense that $l|a| \ll R$. The shifted mean radius $\hat{R}$ is implicitly defined by the requirement that the perturbed interface be a Lagrangian surface across which no mass or volume is transported. This requirement may be expressed as $\int d \Omega \hat{r}^{3}=4 \pi R^{3}$, where $d \Omega=\sin \theta d \theta d \phi$ $=2 \pi \sin \theta d \theta$. Since $a$ is small, however, it is unnecessary to satisfy this requirement exactly, but in the present context we must satisfy it to second order in $a$ for reasons to be explained below. Solving for $\hat{R}$ to second order in $a$, we obtain

$$
\hat{R}=R\left[1-\frac{1}{2}\left(\frac{a}{R}\right)^{2}\right],
$$

where use has been made of the well-known relations

$$
\begin{gathered}
\int d \Omega P_{l}(\cos \theta)=0, \\
\int d \Omega P_{l}^{2}(\cos \theta)=\frac{4 \pi}{2 l+1} .
\end{gathered}
$$

Equations (3) and (4), together with the easily verified relation

$$
\int d \Omega\left(\frac{\partial P_{l}}{\partial \theta}\right)^{2}=\frac{4 \pi l(l+1)}{2 l+1}
$$

will also be needed in what follows. 
By writing Eq. (1), we have restricted attention to perturbations with no dependence on the azimuthal angle $\phi$, which greatly simplifies the analysis. In a more general treatment, the Legendre polynomial $P_{l}(\cos \theta)$ would be replaced by a spherical harmonic $Y_{l}^{m}(\theta, \phi)(|m| \leqslant l)$, which becomes proportional to $P_{l}(\cos \theta)$ for $m=0$. Fortunately, however, the restriction to $m=0$ entails no real loss in generality, as it is known from previous linear studies that the perturbation growth rate is independent of the azimuthal mode number $m$ [4-6]. We shall heuristically assume that the same remains true in the nonlinear regime as well. Direct numerical simulations provide some limited support for this assumption [6].

We require the potential flow field $\mathbf{u}=\nabla \Phi$ that results from the perturbed interface motion to first order in $a$. The potential $\Phi$ has been determined by Plesset [3], and is given by $\Phi=\Phi_{1}$ for $r<\hat{r}$ and $\Phi=\Phi_{2}$ for $r>\hat{r}$, where

$$
\begin{aligned}
\Phi_{1}=-\frac{R^{2} \dot{R}}{r} & +\frac{1}{l}\left(\frac{2 l+1}{2}\right)^{1 / 2}(R \dot{a}+2 \dot{R} a)\left(\frac{r}{R}\right)^{l} P_{l}(\cos \theta), \\
\Phi_{2}= & -\frac{R^{2} \dot{R}}{r}-\frac{1}{l+1}\left(\frac{2 l+1}{2}\right)^{1 / 2}(R \dot{a}+2 \dot{R} a) \\
& \times\left(\frac{R}{r}\right)^{l+1} P_{l}(\cos \theta),
\end{aligned}
$$

and $\dot{q}=d q / d t$ for any quantity $q$. The total kinetic energy of the system is given by $T=T_{1}+T_{2}$, where

$$
\begin{aligned}
& T_{1}=\frac{\rho_{1}}{2} \int d \Omega \int_{R_{1}}^{\hat{r}} r^{2} d r\left|\nabla \Phi_{1}\right|^{2}, \\
& T_{2}=\frac{\rho_{2}}{2} \int d \Omega \int_{\hat{r}}^{R_{2}} r^{2} d r\left|\nabla \Phi_{2}\right|^{2} .
\end{aligned}
$$

Since $T$ is quadratic in $\Phi, T_{1}$ and $T_{2}$ must be evaluated to second order in $a$ and/or $\dot{a}$ to describe the linear regime. For this purpose it is essential to consistently retain all secondorder terms arising from $\hat{r}$ in Eqs. (8) and (9), and this is why it was necessary to evaluate $\hat{R}$ to second order in $a$. Just as in the planar case [1], however, the linearized equations (6) and (7) for $\Phi_{1}$ and $\Phi_{2}$ are nevertheless sufficient to determine $T_{1}$ and $T_{2}$ to second order, since the linearized interface dynamics is completely determined by the linear approximation to $\Phi$ [3]. The second-order corrections to $\Phi_{1}$ and $\Phi_{2}$ therefore cannot contribute to $T_{1}$ and $T_{2}$ to second order, and this has been directly confirmed by a more detailed analysis. Thus $T$ may be determined to second order by combining Eqs. (6)(9), (1), and (2), expanding the results to second order in $a$ and/or $\dot{a}$, and making use of Eqs. (3)-(5) as needed. This is tedious but straightforward, with the final result

$$
T=T_{0}+2 \pi\left(2 \rho_{l}-\Delta \rho\right) R \dot{R} a(R \dot{a}+\dot{R} a)+\pi \rho_{l} R^{3} \dot{a}^{2},
$$

where

$$
T_{0}=2 \pi R^{4} \dot{R}^{2}\left[\rho_{1}\left(\frac{1}{R_{1}}-\frac{1}{R}\right)+\rho_{2}\left(\frac{1}{R}-\frac{1}{R_{2}}\right)\right]
$$

is the kinetic energy of the unperturbed system, $\Delta \rho \equiv \rho_{2}$ $-\rho_{1}, \quad \rho_{l} \equiv \rho_{1} / l+\rho_{2} /(l+1)$, and small terms of order $\left(R_{1} / R\right)^{2 l+1}$ and $\left(R / R_{2}\right)^{2 l+1}$ have been neglected.

Just as in the planar case, the volume per unit area of the mixing layer is proportional to $a$. In the spherical case, however, the area itself is no longer constant but is proportional to $R^{2}$, which changes with time. Thus the volume of the mixing layer, which is a measure of the amount or degree to which the two fluids have been mixed at any given time, is proportional to $s=R^{2} a$, and a simpler and more fundamental description is obtained by eliminating $a$ and $\dot{a}$ in favor of $s$ and $\dot{s}=R(R \dot{a}+2 \dot{R} a)$. Indeed, Eqs. (6) and (7) show that the perturbation to $\Phi$ is simply proportional to $\dot{s}$, so that the velocity field remains unperturbed when $\dot{s}=0$, even though $a$ and $R$ may be changing with time. The inverse relation between $(a, \dot{a})$ and $(s, \dot{s})$ is given by $a=s / R^{2}$ and $\dot{a}$ $=R^{-3}(R \dot{s}-2 \dot{R} s)$, which may be used to reexpress $T$ in terms of $s$ and $\dot{s}$. We thereby obtain

$$
T=T_{0}+\frac{\pi}{R^{3}}\left[2 \Delta \rho \dot{R} s(\dot{R} s-R \dot{s})+\rho_{l} R^{2} \dot{s}^{2}\right] .
$$

Equation (12) will be used to obtain the dynamical evolution of the interface from Lagrange's equations [2] in terms of the generalized coordinates $s$ and $R$ and their time derivatives $\dot{s}$ and $\dot{R}$. For this purpose we must also consider the potential energy $V$ associated with whatever external forces (presumed conservative) are employed to produce the mean interface motion $R(t)$. But these forces are applied at the surfaces $r$ $=R_{2}$ and $/$ or $r=R_{1}$, so they are independent of $s$. It follows that $V=V(R)$ is also independent of $s$, and will therefore not contribute to the Lagrange equation of motion for $s$ [2]. Since this is the only equation of motion we shall consider, $V(R)$ can henceforth be ignored and the Lagrangian $L$ can simply be identified with $T$.

\section{LINEAR REGIME}

We first verify that this approach correctly reproduces the correct linearized equation of motion for $a$, which was first derived by Plesset [3]. In the absence of dissipation, Lagrange's equation for $s$ is given by [2]

$$
\frac{d}{d t}\left(\frac{\partial T}{\partial \dot{s}}\right)=\frac{\partial T}{\partial s}
$$

Combining Eqs. (12) and (13), and simplifying the result, we obtain

$$
\rho_{l}(R \ddot{s}-\dot{R} \dot{s})-\Delta \rho \ddot{R} s=\rho_{l} R^{2} \frac{d}{d t}\left(\frac{\dot{s}}{R}\right)-\Delta \rho \ddot{R} s=0 .
$$

When $s, \dot{s}$, and $\ddot{s}$ are eliminated in favor of $a$, $\dot{a}$, and $\ddot{a}$, this equation reduces to precisely the linearized equation of motion for $a$ previously derived by Plesset [3]. Notice that Eq. (14) admits the solution $s=$ const when $\Delta \rho=0$, so that a perturbation with $\dot{s}=0$ initially produces no further mixing when the fluids have the same density. This does not, however, imply that $\dot{a}=0$ in this case. In particular, if $R$ decreases then $a$ increases for purely geometrical reasons, but this is a mere squeezing or stretching effect which does not 
transport any additional mass of either fluid across the surface $r=R$, and hence does not represent true mixing. (It does, however, change the surface area of the perturbed interface. This in turn will alter the rate of mixing due to molecular diffusion, but the model in its present form neglects this effect.)

\section{NONLINEAR REGIME}

In contrast to the planar case, the perturbation is not sinusoidal in the present context and consequently has no welldefined wavelength. However, it is nevertheless convenient to define the effective wavelength of the perturbation to be twice the mean distance between nodes; i.e.,

$$
\lambda=\frac{2 \pi R}{l}
$$

or $l=2 \pi R / \lambda$. In the linear regime, $l$ is simply constant with its initial value $l_{0}$. Just as in the planar case, we shall extend Eq. (12) into the nonlinear regime by means of a wavelength renormalization hypothesis (WRH) [1], according to which $\lambda$ is continuously dynamically renormalized to a value of order $|a|$. The rationale for the WRH was discussed in detail in Ref. [1]. In contrast to the planar case [1], Eq. (15) shows that $\lambda$ is not constant in the linear regime but varies with $R$. The WRH introduces an additional dependence on $a$ or $s$ in the nonlinear regime, so that $\lambda=\lambda(R, s)$ in general, a form which subsumes the linear regime as a special case. It then follows from Eq. (15) that $l$ and $\rho_{l}$ are no longer constants but are now replaced by $l(R, s)=2 \pi R / \lambda(R, s)$, and $\rho_{l}(R, s)=\rho_{1} / l(R, s)+\rho_{2} /[l(R, s)+1]$. These replacements and functional dependences will be understood in what follows. A provisional form for the function $\lambda(R, s)$ will be proposed in Sec. VI based on the form of $\lambda(|a|)$ used in the planar case.

As discussed in Ref. [1], it is necessary to allow for energy dissipation in the nonlinear regime. This can be done by introducing an additional generalized force $Q$ into Lagrange's equation of motion for $s(t)$, which then takes the form [2]

$$
\frac{d}{d t}\left(\frac{\partial T}{\partial \dot{s}}\right)=\frac{\partial T}{\partial s}+Q
$$

The dissipative force $Q$ will be taken to be a natural spherical analog of the form used in the planar case [1], namely,

$$
Q=-4 \pi c \bar{\rho} \frac{|\dot{s}| \dot{s}}{R^{4}}
$$

where $2 \bar{\rho}=\rho_{1}+\rho_{2}$, a factor of $4 \pi R^{2}$ has been inserted to convert from energy per unit area to energy itself, and $c$ $\geqslant 0$ is another dimensionless coefficient of order unity. Note that this expression properly vanishes as it should when $\dot{s}$ $=0$, so that there is no dissipation of energy in the absence of true mixing.

Since Eq. (16) involves no partial derivatives with respect to $R$ or $\dot{R}$, both of which are given functions of time, the presence of $R$ and $\dot{R}$ in $T$ is simply equivalent to an explicit time dependence. Thus $T$ may be regarded as a function of $(s, \dot{s}, t)$, which it is convenient to write in the form

$$
T(s, \dot{s}, t)=A_{0}(s, t)+A_{1}(s, t) \dot{s}+A_{2}(s, t) \dot{s}^{2},
$$

where

$$
\begin{gathered}
A_{0}=T_{0}+2 \pi \Delta \rho \frac{\dot{R}^{2} s^{2}}{R^{3}}, \\
A_{1}=-2 \pi \Delta \rho \frac{\dot{R} s}{R^{2}}, \\
A_{2}=\frac{\pi \rho_{l}}{R} .
\end{gathered}
$$

Combining Eqs. (16) and (18), we obtain

$$
F_{3} \ddot{s}+F_{2} \dot{s}^{2}+F_{1} \dot{s}+F_{0}=Q,
$$

where

$$
\begin{gathered}
F_{0}(s, t)=\left(\frac{\partial A_{1}}{\partial t}\right)_{s}-\left(\frac{\partial A_{0}}{\partial s}\right)_{t}=-2 \pi \Delta \rho \frac{\ddot{R} s}{R^{2}} \\
F_{1}(s, t)=2\left(\frac{\partial A_{2}}{\partial t}\right)_{s}=\frac{2 \pi \dot{R}}{R^{2}}\left(R \frac{\partial \rho_{l}}{\partial R}-\rho_{l}\right), \\
F_{2}(s, t)=\left(\frac{\partial A_{2}}{\partial s}\right)_{t}=\frac{\pi}{R} \frac{\partial \rho_{l}}{\partial s}, \\
F_{3}(s, t)=2 A_{2}=\frac{2 \pi \rho_{l}}{R} .
\end{gathered}
$$

Combining Eqs. (17) and (22)-(26) and simplifying, we finally obtain

$$
2 R^{2} \frac{d}{d t}\left(\frac{\rho_{l} \dot{s}}{R}\right)-\left(\frac{\partial \rho_{l}}{\partial s}\right) R \dot{s}^{2}-2 \Delta \rho \ddot{R} s+4 c \bar{\rho} \frac{|\dot{s}| \dot{s}}{R^{2}}=0 .
$$

Equation (27) is the fundamental dynamical evolution equation of the model. It is a second-order ordinary differential equation which determines $s(t)$ for an arbitrary given $R(t)$. Notice that like the linear equation (14), it properly admits the solution $s=$ const when $\Delta \rho=0$. However, the model is not yet complete because we have not yet defined $l(R, s)$. This will be done in Sec. VI.

\section{CONSERVATION PROPERTIES}

In the planar case, a constant interface acceleration is equivalent to a time-independent potential energy in terms of which a conservation law can be established for the intrinsic energy (kinetic plus potential) of the mixing layer [1]. This case is particularly straightforward because there is a clean separation between the energy of the mixing layer and the kinetic energy of the center of mass of the system. The spherical case does not appear to admit such a separation, and its conservation properties are consequently less straightforward. Of course, the Lagrangian formulation still guaran- 
tees that the total energy of the system, including $V(R)$, is conserved in the absence of dissipation. In the spherical case, however, this energy does not appear to separate naturally into a well-defined center-of-mass energy and a physically significant remainder as it does in the planar case.

In spite of this, a spherical analog of the planar conservation law can still be established. Like the planar law, this spherical analog is a reflection and consequence of total energy conservation, but it is somewhat more artificial and does not possess the same clear physical interpretation. However, it nevertheless represents a true conservation property of the model equations, and as such it seems desirable to preserve it when solving these equations numerically. We shall utilize it for this purpose in Sec. VIII below.

We proceed by specializing Eq. (27) to the case of zero dissipation $(c=0)$ and multiplying by $\dot{s} / R$ to obtain

$$
\frac{d}{d t}\left(\frac{\rho_{l} \dot{s}^{2}}{R^{2}}\right)+\left(\dot{R} \frac{\partial \ln \rho_{l}}{\partial R}\right)\left(\frac{\rho_{l} \dot{s}^{2}}{R^{2}}\right)=\Delta \rho \frac{\ddot{R}}{R^{3}} \frac{d}{d t}\left(s^{2}\right) .
$$

We now observe that if $\rho_{l}(R, s)$ is of the factored form $\rho_{l}$ $=f(R) g(s)$, then

$$
\dot{f}=\left(\dot{R} \frac{\partial \ln \rho_{l}}{\partial R}\right) f
$$

so that Eq. (28) becomes

$$
\frac{d}{d t}\left(\frac{f \rho_{l} \dot{s}^{2}}{R^{2}}\right)=f \Delta \rho \frac{\ddot{R}}{R^{3}} \frac{d}{d t}\left(s^{2}\right) .
$$

This shows that when the implosion history $R(t)$ is such that $f(R) \ddot{R} / R^{3}$ is constant, the quantity

$$
E=f\left(\frac{\rho_{l}}{R^{2}} \dot{s}^{2}-\frac{\Delta \rho \ddot{R}}{R^{3}} s^{2}\right)
$$

is conserved in the motion defined by Eq. (27) with $c=0$; i.e., $\dot{E}=0$.

Unfortunately, $\rho_{l}$ cannot in general be assumed to have the factored form upon which this conservation law depends. However, this law can be formally preserved in general by the simple artifice of regarding $f$ as a function of $t$ defined by Eq. (29) rather than as a function of $R$ related directly to $\rho_{l}$. With this reinterpretation, $E$ is still conserved when $f(t) \ddot{R} / R^{3}$ is constant.

\section{WAVELENGTH RENORMALIZATION HYPOTHESIS}

In the planar case [1], the effective wavelength $\lambda$ of the perturbed interface was taken to be of the form

$$
\lambda=\max \left(\lambda_{0}, b|a|+(1-m b) \lambda_{0}\right),
$$

where $\lambda_{0}$ is the wavelength of the initial perturbation, $b$ is a dimensionless proportionality constant, and $m \sim 1$ is a parameter which specifies the amplitude-to-wavelength ratio at which the transition to the nonlinear regime occurs. Since the behavior of the spherical case is much more rich and subtle than the planar case, there is as yet no assurance that a simple prescription of this form will be adequate in the present context. In the absence of other information, however, it seems reasonable to employ Eq. (32) on a provisional basis in the spherical case as well, with the understanding that $\lambda_{0}$ is no longer a constant but now varies with $R$ according to Eq. (15), so that $\lambda_{0}=2 \pi R / l_{0}$. Equation (15) then implies that $l$ is given by

$$
l=l_{0}\left[1-m b+b \max \left(m, \frac{l_{0}|s|}{2 \pi R^{3}}\right)\right]^{-1} .
$$

This completes the model except for the choice of $b, c$, and $m$, which will be discussed in Sec. VII.

We note that in this spherical version of the WRH, $l$ (and hence $\rho_{l}$ and $\lambda$ as well) does not depend on $R$ and $s$ separately but only on the composite variable $s / R^{3}$, just as one would expect on dimensional grounds. We further note that $l$ has now become a continuous variable and is no longer an integer. Thus the spherical WRH requires the heuristic analytic continuation, as it were, of $l$ from discrete to continuous values, in marked contrast to the planar case where $\lambda$ is continuous from the outset. This seems harmless, however, since $\lambda$ becomes a somewhat nebulous effective wavelength in the nonlinear regime [1], so that the corresponding value of $l$ no longer literally represents the mode number of a single Legendre polynomial as it does in the linear regime. Allowing $l$ to vary continuously presents no problems, as it enters into the model only through $\rho_{l}$, which is a smooth continuous function of $l$. Of course, it is easier to visualize $l$ as a continuous variable when $l \gg 1$. Equation (15) shows that this is indeed the case as long as $\lambda \ll R$, and if this condition were seriously violated one would intuitively expect the accuracy of the model to deteriorate in any case.

\section{SPECIAL CASES}

In the linear regime with zero dissipation, $c=0$ and $l$ $=l_{0}$, so $\partial \rho_{l} / \partial s=0$, and Eq. (27) immediately reduces to Eq. (14). It is also of interest to examine the behavior of the model as $R \rightarrow \infty$, where it would intuitively be expected to reduce to the analogous planar model developed in Ref. [1]. In this limit we have $\dot{s} \rightarrow R^{2} \dot{a}$ and $\ddot{s} \rightarrow R^{2} \ddot{a}$. However, for this limit to be sensible it must be taken in such a way that $\lambda$ remains finite, which requires that we simultaneously send $l \rightarrow \infty$ at a finite ratio $l / R=2 \pi / \lambda$ [4,5]. It follows that $\rho_{l}$ $\rightarrow 2 \bar{\rho} / l=\bar{\rho} \lambda /(\pi R)$ and $\partial \rho_{l} / \partial s \rightarrow R^{-3}(\bar{\rho} / \pi)(\partial \lambda / \partial a)$. Combining the above relations with Eq. (27), we obtain precisely Eq. (13) of Ref. [1]. This confirms that the present spherical model reduces to the corresponding planar model as $R$ $\rightarrow \infty$. Since this reduction occurs with no redefinition of the model coefficients $b$ and $c$, it seems reasonable in the absence of other information to set $b$ and $c$ to the same values used in the planar case, namely [1],

$$
\begin{gathered}
b=\frac{\pi \theta}{\alpha(2-\theta)}, \\
c=\frac{2-3 \theta}{4 \alpha(2-\theta)},
\end{gathered}
$$

where $\alpha$ and $\theta$ are experimentally accessible parameters appearing in the late-time scaling laws for the planar Rayleigh- 
Taylor (RT) and Richtmyer-Meshkov (RM) instabilities, respectively [1]. Similarly, it seems reasonable to provisionally set $m$ to whatever value is used in the planar case. However, since the form of the dissipation term $Q$ was obtained from inherently nonlinear considerations [1], this term should be switched off in the linear regime by setting $c=0$ when $l$ $=l_{0}$.

\section{NUMERICAL SOLUTION OF THE MODEL EQUATIONS}

In general it will be necessary to solve Eq. (27) numerically to obtain solutions for arbitrary implosion histories $R(t)$. For numerical purposes it is convenient to replace the second-order equation (27) by an equivalent system of two coupled first-order equations. It is further desirable to choose a numerical scheme which preserves the conservation properties established in Sec. V. To this end we define the new variable $w=\sqrt{f \rho_{l}} \dot{s} / R$, so that

$$
\dot{s}=\frac{R w}{\sqrt{f \rho_{l}}},
$$

and Eq. (27) then becomes

$$
\dot{w}=\sqrt{\frac{f}{\rho_{l}}} \frac{\Delta \rho \ddot{R} s}{R^{2}}-\frac{2 c \bar{\rho}|w| w}{R^{2} \sqrt{f \rho_{l}^{3}}},
$$

where $f=f(t)$ is still defined by Eq. (29). It is then easy to verify that the following numerical scheme preserves the conservation properties established in Sec. V:

$$
\begin{gathered}
\frac{s^{n+1}-s^{n}}{\Delta t}=\left(\frac{R}{2 \sqrt{f \rho_{l}}}\right)^{n}\left(w^{n+1}+w^{n}\right), \\
\frac{w^{n+1}-w^{n}}{\Delta t}=\left(\sqrt{\frac{f}{\rho_{l}}} \frac{\Delta \rho \ddot{R}}{2 R^{2}}\right)^{n}\left(s^{n+1}+s^{n}\right)-\left(\frac{2 c \bar{\rho}|w|}{R^{2} \sqrt{f \rho_{l}^{3}}}\right)^{n} w^{n+1} .
\end{gathered}
$$

Here $q^{n}$ denotes the numerical approximation to the quantity $q$ at time $t^{n}$, and $\Delta t=t^{n+1}-t^{n}$ is the time step. This numerical scheme has the same essential structure as that used in the planar case [1]. The most important natural time scale $\tau$ in these equations is given by $1 / \tau^{2}=\left|\left(\Delta \rho / \rho_{l}\right)(\ddot{R} / R)\right|$, and it is of course necessary to restrict $\Delta t \ll \tau$ to obtain an accurate solution. Equations (38) and (39) constitute a linear system of two equations in the two unknown quantities $s^{n+1}$ and $w^{n+1}$. These equations are easily solved to advance the system in time.

Of course, the use of this scheme also requires numerical solution of the auxiliary equation (29) in order to determine $f$. For this purpose the following scheme seems suitable:

$$
\frac{f^{n+1}-f^{n}}{\Delta t}=\frac{1}{2}\left(\dot{R} \frac{\partial \ln \rho_{l}}{\partial R}\right)^{n+1}\left[(1+\sigma) f^{n}+(1-\sigma) f^{n+1}\right],
$$

where $\sigma$ is the sign of $\left(\dot{R} \partial \ln \rho_{l} / \partial R\right)^{n+1}$.

The above numerical scheme has been used to calculate the nonlinear perturbation growth during two spherical im-

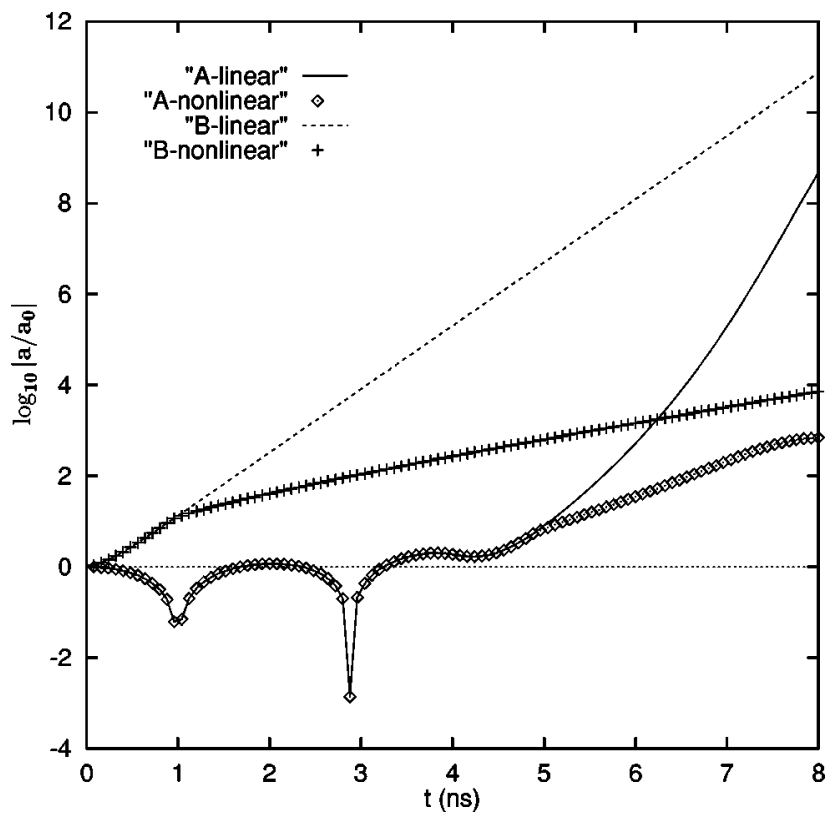

FIG. 1. Perturbation amplitude vs time for Mikaelian cases $A$ and $B$.

plosion histories for which the linear growth has previously been studied by Mikaelian [5]. In these calculations, a spherical interface with a density ratio of $\rho_{2} / \rho_{1}=10$ and an initial radius of $R_{0}=2.5 \mathrm{~mm}$ is imploded to a final radius of $0.1 \mathrm{~mm}$ over a time interval of $8 \mathrm{~ns}$. The initial perturbation mode number is $l_{0}=50$, and the initial perturbation amplitude was arbitrarily taken to be $a_{0}=10^{-2} \mathrm{~mm}$. (In the linear case, the actual values of $a$ and $a_{0}$ are immaterial, since only the ratio $a / a_{0}$ is significant. However, this is no longer the case in the nonlinear model, where the value of $a_{0}$ affects the transition to the nonlinear regime.) The RT and RM scaling parameters were taken to be $\alpha=0.061$ and $\theta=0.37$ [7], and $m$ was taken to be 0.5 .

The two implosion histories considered by Mikaelian were a constant inward acceleration followed by a constant deceleration (case $A$ ), and an exponential implosion (case $B)$. In case $A, \ddot{R}=-150 \mu \mathrm{m} / \mathrm{ns}^{2}$ for $0<t<4 \mathrm{~ns}$ and $\ddot{R}$ $=150 \mu \mathrm{m} / \mathrm{ns}^{2}$ for $4 \mathrm{~ns}<\mathrm{t}<8 \mathrm{~ns}$. In case $B, R=R_{0} e^{t / T}$, where $T=-8 / \ln 25 \mathrm{~ns}$. Plots of $\log _{10}\left|a / a_{0}\right|$ vs time for both cases are shown in Fig. 1. Also shown for comparison purposes are the purely linear growth curves, which agree with those presented in Mikaelian's Fig. 3 [5]. As expected, the perturbation growth rates slow considerably after the transition to the nonlinear regime, corresponding to the decrease in the effective mode number with the growth of the mixing region.

As previously discussed, the degree of true mixing is proportional to $s$ rather than $a$, so inspection of $a(t)$ alone is liable to be misleading. The corresponding plots of $\log _{10}\left|s / s_{0}\right|$ vs time are therefore given in Fig. 2, which shows that in spite of the growth in perturbation amplitude, no significant true mixing occurs in the nonlinear regime in either of these cases.

Finally, to obtain some insight into the effect of the spherical geometry, we performed corresponding planar calculations with the same $a_{0}, \lambda_{0}, \dot{R}(t)$, and $\ddot{R}(t)$, but with $R_{0}$ set to a very large value to reach the planar limit. The resulting plots of $\log _{10}\left|a / a_{0}\right|$ vs time are shown in Fig. 3. Com- 


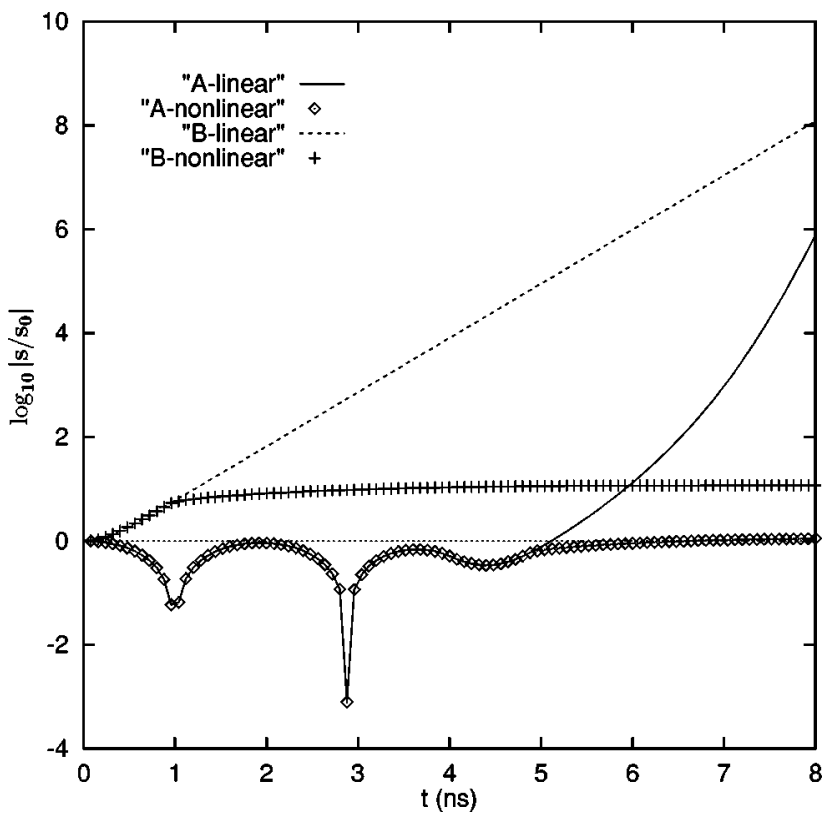

FIG. 2. Perturbation volume vs time for Mikaelian cases $A$ and $B$.

parison with Fig. 1 shows that in these particular cases, the spherical geometry enhances the linear growth rates by many orders of magnitude, while the final nonlinear perturbation amplitudes are also enhanced but to a much lesser degree. We reemphasize, however, that the perturbation amplitude $a$ alone does not provide an adequate measure of the degree of mixing in spherical problems with significant changes in $R$.

\section{CONCLUSION}

We have presented a simple model, embodied in Eqs. (27) and (33), for predicting the time evolution of an incompressible spherical fluid mixing layer subjected to an arbitrary time-dependent implosion history $R(t)$. It is hoped that this model will provide a useful tool for making predictive estimates of mixing at unstable fluid interfaces in spherical geometry with variable implosion histories. Of course, the accuracy and utility of the model can only be assessed by comparisons with data from experiments and/or threedimensional direct numerical simulations. (Two-dimensional simulations would be suggestive but not definitive, since the nonlinear dynamics of the mixing layer is fully threedimensional regardless of the dimensionality of the initial perturbations.) Unfortunately, suitable data of this type do not yet seem to be available. However, the model was con-

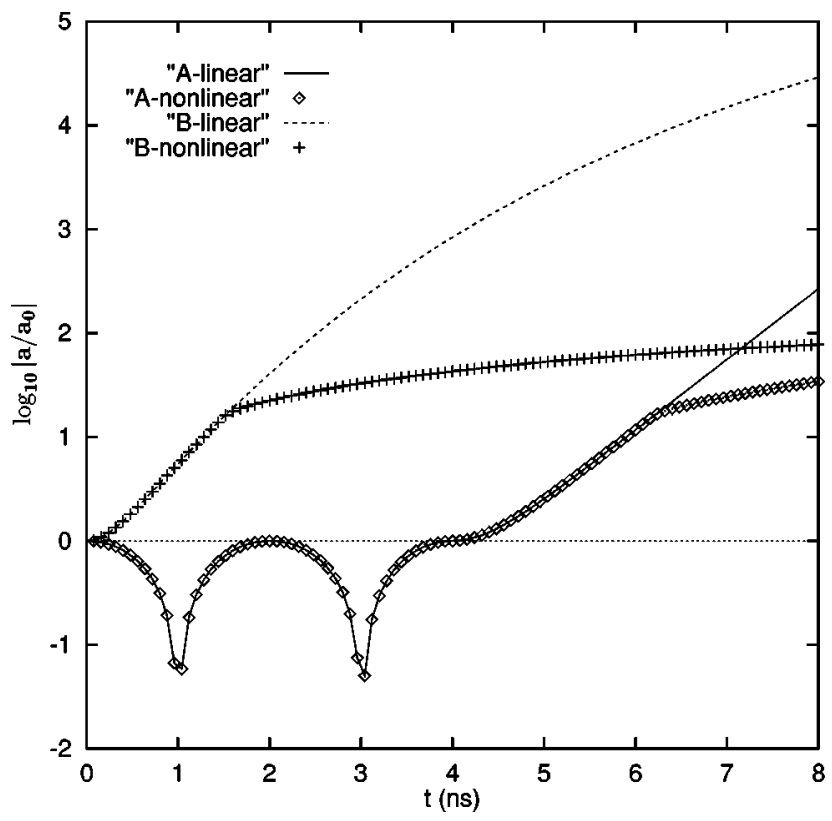

FIG. 3. Perturbation amplitude vs time for planar Mikaelian cases $A$ and $B$.

structed by the same procedure used to obtain the corresponding planar model [1], which has been shown to reproduce correctly all of the known growth laws and scaling behavior for both the Rayleigh-Taylor and RichtmyerMeshkov instabilities in both the linear and nonlinear regimes. Moreover, the model correctly reduces to the Plesset equation [3] in the linear regime and to the corresponding planar model [1] in the limit $R \rightarrow \infty$. This lends cause for optimism, and perhaps warrants the use of the model on a provisional basis until such time as proper validation studies can be performed. Just as in the planar case, however, we emphasize that application of the model to compressible fluids with shocks will require one to distinguish between and correct for differences in the preshock and post-shock conditions, particularly densities and perturbation amplitude. These corrections have not been considered here, but they are of essential importance for many practical applications.

\section{ACKNOWLEDGMENTS}

I am grateful to Karnig Mikaelian and Oleg Schilling for helpful discussions, and for directing my attention to some of the relevant literature. This work was performed under the auspices of the U.S. Department of Energy by Lawrence Livermore National Laboratory under Contract No. W-7405ENG-48.
[1] J. D. Ramshaw, Phys. Rev. E 58, 5834 (1998).

[2] H. Goldstein, Classical Mechanics, 2nd ed. (Addison-Wesley, Reading, MA, 1980).

[3] M. S. Plesset, J. Appl. Phys. 25, 96 (1954).

[4] K. Mikaelian, Phys. Rev. Lett. 65, 992 (1990).
[5] K. Mikaelian, Phys. Rev. A 42, 3400 (1990).

[6] H. Sakagami and K. Nishihara, Phys. Rev. Lett. 65, 432 (1990).

[7] G. Dimonte and M. Schneider, Phys. Rev. E 54, 3740 (1996). 